\title{
Lattice Gauge Theory and the Origin of Mass*
}

\author{
Andreas S. Kronfeld \\ Theoretical Physics Department, Fermi National Accelerator Laboratory, \\ Batavia, Illinois, USA
}

\begin{abstract}
Most of the mass of everyday objects resides in atomic nuclei; the total of the electrons' mass adds up to less than one part in a thousand. The nuclei are composed of nucleons - protons and neutrons - whose nuclear binding energy, though tremendous on a human scale, is small compared to their rest energy. The nucleons are, in turn, composites of massless gluons and nearly massless quarks. It is the energy of these confined objects, via $M=E / c^{2}$, that is responsible for everyday mass. This article discusses the physics of this mechanism and the role of lattice gauge theory in establishing its connection to quantum chromodynamics.
\end{abstract}

\section{Introduction}

With the recent observation of a Higgs-like particle, $\frac{1+3}{3}$ people from all walks of life are talking about the origin of mass. Careful accounts note that this new object's underlying field generates mass neither for luminous matter nor for dark matter but for standard-model particles. Among these, the top quark and the $W$ and $Z$ bosons are especially intriguing, the storyline goes, because their masses are similar to those of whole atoms of gold or silver. But where does the mass of a gold ring or a silver spoon come from? This article reviews our understanding of the origin of mass of these and all other everyday objects, starting from first principles.

The density of gold metal is around $20 \mathrm{~g} \mathrm{~cm}^{-3}$. At the beginning of the twentieth century, no one knew how mass is distributed within atoms, and several ideas had been put forth 45 Then an experiment carried out by Hans Geiger and Ernest Marsden found an astonishing rate of wide-angle scattering of a beam $\alpha$ particles incident on a gold foil ${ }^{6}$ Their laboratory director, New Zealander Ernest Rutherford, realized that their findings could be understood if atoms contain a massive nucleus surrounded by a cloud of electrons.$^{7}$ The density of nuclear material ranges from $20 \times 10^{13} \mathrm{~g} \mathrm{~cm}^{-3}$ for a gold nucleus to $60 \times 10^{13} \mathrm{~g} \mathrm{~cm}^{-3}$ for an isolated proton. One cubic millimeter (the size of a coarse grain of sand) of such nuclear material weighs about as much as two aircraft carriers.

The discovery of the neutron ${ }^{8}$ showed that atomic nuclei consist of protons and neutrons, bound together by the so-called strong force. The forces in the nucleus

*Prepared for One Hundred Years of Subatomic Physics, edited by Ernest Henley and Stephen Ellis. 
generate tremendous energy, yet nuclear fission releases only around one part in a thousand of the total rest energy. Nuclear fusion producing ${ }^{4} \mathrm{He}$ releases a larger fraction of the total nuclear rest energy, but still less than $1 \%$. Thus, the origin of the bulk of nuclear mass lies beyond nuclear chemistry and more deeply within the nucleons themselves.

Of course, the nucleon has structure too. Indeed, deeply inelastic electronnucleon scattering measurements (wide angles again) are modeled well with weakly interacting constituents known as partons $9[10$ To obtain a full appreciation of the interior of the nucleon, however, one must to turn to the modern theory of the strong interactions, namely quantum chromodynamics (QCD). QCD merges the ideas of the quark model (introduced to account for the plethora of stronglyinteracting hadrons 11 13), the quantum number "color" (introduced to reconcile spin and statistics 1415 ), and partons into a self-contained theory 16

The Lagrangian of $\mathrm{QCD}^{177}$ looks like that of quantum electrodynamics (QED). In both cases, the interactions are specified by a gauge symmetry, SU(3) for QCD and $\mathrm{U}(1)$ for QED. $\mathrm{SU}(N)$ is the nonabelian group of $N \times N$ unitary matrices with unit determinant. As a consequence of the nonabelian, i.e., noncommuting, nature of $\mathrm{SU}(N)$, the quanta of the gauge field - known in QCD as gluons - carry color $18 / 19$ Because QED's U(1) group of phase factors commutes, the gauge quantum is electrically neutral, in accord with the natural behavior of the photon. The self-coupling of the gluon is responsible for the markedly different dynamics in QCD. In particular, quantum effects, which can be examined in one-loop perturbation theory, render the QCD coupling smaller and smaller at short distances 20|21 This "asymptotic freedom" means that QCD reproduces the simplicity of the parton model.

The flip side of asymptotic freedom is that the strong interaction strengthens at large distances. A "typical mass scale $\Lambda_{\mathrm{QCD}}$ " separates weak from strong coupling. At distances large enough so that the coupling is strong, perturbative techniques are insufficient to understand fully what happens. Nevertheless, the strengthening of the force provides a hint that it is possible to explain not only the origin of hadronic mass but also why isolated quarks are never observed (known as confinement). This article discusses how, a century after the Geiger-Marsden experiment, we have established a connection from the QCD Lagrangian to the mass of the atomic nucleus and, hence, all everyday objects. Indeed, this connection sheds light on confinement as well. The central theoretical and conceptual tool is lattice gauge theory,$\sqrt{22}$ which enables nonperturbative calculations via a mathematically rigorous definition of quantum field theory. The calculations lie beyond the scope of pencil and paper and, in fact, rely on leadership-class supercomputers.

The rest of this article is organized into two main parts. Section 2 recalls the early (and prehistoric) development of lattice gauge theory. Section 3 reviews QCD calculations based on lattice gauge theory, with special attention to calculations the shed light on the origin of (everyday) mass. The Appendix recounts a tale about lattice field theory, Werner Heisenberg, and a children's puzzle. 


\section{Lattice Gauge Theory}

Before turning to lattice gauge theory itself, it is helpful to discuss asymptotic freedom a bit more. Let us start with the relation between the bare gauge coupling and a renormalized coupling. A physical renormalization scheme comes from the force $F(r)$ between static source and sink of color, separated by a distance $r$,

$$
r^{2} F(r)=-\frac{C_{F}}{4 \pi} g_{F}^{2}(r),
$$

where $C_{F}=1$ for $\mathrm{U}(1), C_{F}=\left(N^{2}-1\right) / 2 N$ for $\mathrm{SU}(N)$. In perturbation theory, the force arises from one-gluon exchange and from Feynman diagrams with loops. To define the loop integrals, one must have an ultraviolet cutoff. A lattice with spacing $a$ builds one from the outset. Adopting lattice notation, the relation between $g_{F}^{2}$ and the bare coupling $g_{0}^{2}$ can be written as follows:

$$
g_{F}^{-2}(r)=g_{0}^{-2}(a)+\beta_{0} \ln \left(a^{2} / r^{2}\right)+c_{F \leftarrow 0}+\mathrm{O}\left(g^{2}\right) .
$$

The constants $\beta_{0}$ and $c_{F \leftarrow 0}$ stem from the one-loop diagrams, and the omitted terms from diagrams with two or more loops. For what follows, $c_{F \leftarrow 0}$ is not very important, but the sign of $\beta_{0}$ is key. Direct calculation in $\mathrm{SU}(N)$ gauge theories yields $\underline{20 \mid 21}$

$$
\beta_{0}=-\frac{2}{3} \frac{n_{f}}{16 \pi^{2}}+\frac{11}{3} \frac{N}{16 \pi^{2}},
$$

where $n_{f}$ is the number of quark flavors. In QED, the second term, which stems from the gluon loop, is absent, and $n_{f}$ is replaced with $2 \sum_{l} q_{l}^{2}$, where $q_{l}$ is the electric charge of charged particle $l$ (e.g., the electron $q_{e}=-1$ ). In QCD with $n_{f} \leq 16$, one finds $\beta_{0}>0$, which yields asymptotic freedom, namely $g_{F}^{2}(r)$ decreases as $r$ decreases. In QED (and in QCD with $n_{f}>16$ ), $\beta_{0}<0$.

Renormalization of the bare gauge coupling $g_{0}^{2}(a)$ makes the right-hand side of Eq. (2) independent of the lattice spacing $a$. Then one can write

$$
g_{F}^{-2}(r)=\beta_{0} \ln \left(r^{-2} / \Lambda_{F}^{2}\right),
$$

where a scale $\Lambda_{F}$ appears

$$
\Lambda_{F}=a^{-1} e^{-1 / 2 \beta_{0} g_{0}^{2}(a)} e^{-c_{F \leftarrow 0} / 2 \beta_{0}} .
$$

If $\beta_{0}<0$ as in QED, this scale is commensurate with the ultraviolet cutoff. ${ }^{23}$ On the other hand, if $\beta_{0}>0$, as in QCD with the six observed quark flavors, the scale $\Lambda_{F}$ is much smaller than the cutoff. Such hierarchies of scale are an essential feature of renormalization in a more general, nonperturbative context $24 \mid 25$

Different renormalization schemes lead to different scales. ${ }^{26}$ In a scheme " $R$ ",

$$
\Lambda_{R}=\Lambda_{F} e^{-c_{R \leftarrow F} / 2 \beta_{0}},
$$

where $c_{R \leftarrow F}=c_{R \leftarrow 0}-c_{F \leftarrow 0}$ is regulator independent. For small exponents, such scales are quantitatively similar. Qualititatively, the range of such scales marks the transition from weak to strong coupling and is usually called $\Lambda_{\mathrm{QCD}}$. 
The discovery of asymptotic freedom spawned widespread interest in all aspects of QCD, including applications to high-energy scattering processes ${ }^{27}$ as well as puzzles such as the nonobservation of isolated quarks. ${ }^{28}$ Kenneth Wilson, who had been working on critical phenomena, was one of those who (re)directed his attention to the strong interactions. When reading his 1974 paper introducing lattice gauge theory, ${ }^{22}$ it may look as though he developed lattice gauge theory to study confinement. In 2004, however, he reminisced ${ }^{29}$

The discovery of asymptotic freedom, made possible by earlier developments on the renormalizability of non-Abelian gauge theories by Veltman and 't Hooft,, $30 \mid 31]$ made it immediately clear, to me as well as many others, that the preferred theory of strong interactions was [QCD]....

Unfortunately, I found myself lacking the detailed knowledge and skills required to conduct research using renormalized non-Abelian gauge theories. My research prior to 1973 had not required this knowledge so I had never spent the time necessary to acquire it.

What was I to do, especially as I was eager to jump into this research with as little delay as possible? I realized that from my prior work in statistical mechanics I knew a lot about working with lattice theories, including the construction of high temperature expansions for such theories. I decided I might find it easier to work with a lattice version of QCD than with the existing continuum formulation of this theory. Moreover, this meant I could be doing original research immediately, rather than having to spend weeks or months absorbing other people's research.

In gauge theories, the "high-temperature expansion" of statistical mechanics develops a strong-coupling series in powers of $1 / g_{0}^{2}$.

Wilson's 1974 paper ${ }^{22}$ showed how to preserve local gauge invariance when spacetime is replaced with a lattice. The main mathematical ingredient is straightforward. Matter fields transform under local gauge transformations as

$$
\phi(x) \mapsto g(x) \phi(x),
$$

where $g(x)$ is an element of a Lie group $G$, e.g., $\mathrm{U}(1)$ or $\mathrm{SU}(N)$. It is not hard to show that the so-called parallel transporter

$$
U_{s}(x, y)=\mathbb{P}_{s} \exp \int_{x}^{y} d z \cdot A(z)
$$

transforms as

$$
U_{s}(x, y) \mapsto g(x) U_{s}(x, y) g^{-1}(y) .
$$

Here $A^{\mu}(x)$ is the gauge potential, taking values in the Lie algebra of $G$. The pathordering symbol $\mathbb{P}_{s}$ prescribes the order of matrix factors in the power series of the exponential function to lie along the path $s$ from $x$ to $y$. From Eqs. (7) and (9),

aWilson's work in statistical mechanics started out as an application of his renormalization-group ideas from particle physics $24 \mid 25$ to critical phenomena $32 \mid 33$ It was very successful, leading to a renormalization-group solution of the Kondo problem of magnetic impurities in nonmagnetic metals 34 that earned him the 1982 Nobel Prize in Physics 35 
products of the form $\phi^{\dagger}(x) U(x, y) \phi(y)$ clearly are gauge invariant. On a lattice, any $U(x, y)$ can be built out of one-link parallel transporters $U\left(x, x^{\prime}\right)$, where $x$ and $x^{\prime}$ are nearest-neighbor lattice sites. The dynamical variables of lattice gauge theory are, thus, matter fields on sites and gauge-group variables on nearest-neighbor links. Note that this construction works for a lattice of any geometry $[36$

Wilson was not the first to consider lattice gauge theory. Wilson knew $\frac{35}{35}$ about work on lattice field theories by Gregor Wentze $\mathrm{l}^{37}$ and by Leonard Schiff ${ }^{38}$ for the strongly-coupled meson-nucleon system. He did not know, until later $\frac{39}{29}$ about the Ising gauge theory of Franz Wegner $\stackrel{40}{\frac{40}{0}}$ or about the (unpublished) nonabelian lattice gauge constructions of Jan Smit ${ }^{41}$ and of Alexander Polyakov ${ }^{43}$

Wentzel's and Schiff's lattice field theories can be traced, via their textbooks, 4445 back to Werner Heisenberg and Wolfgang Pauli's first paper on the quantization of electrodynamics. To lend rigor to several (then brand new) mathematical manipulations, they introduced a spatial lattice, writing: 46

In der Tat kann man den Fall kontinuierlich vieler Freiheitsgrade, wo die Zustandsgrößen Raumfunktionen sind, stets durch Grenzübergang aus dem Fall endlich vieler Freiheitsgrade gewinnen.

Indeed, one can always obtain the case of continuously many degrees of freedom, where the state variables are functions of space, through a limit of the case of finitely many degrees or freedom. (Author's translation.)

Heisenberg and Pauli thus introduce fields on a discrete set of cells, whose centers form a lattice, and use the limit of smaller and smaller cells to establish the functional equations of motion from their discrete counterpart. They further employ the lattice to derive the Dirac $\delta$ function in equal-time commutators in field theory from the Kronecker $\delta$ symbol in quantum mechanics for a finite collection of degrees of freedom. They do not revisit the lattice when considering local gauge symmetry, so they did not need Eqs. (7)-(9). They also did not discuss electron fields on the lattice - as we shall see below, prudently. Heisenberg later pondered the lattice as the fundamental structure of space, as discussed in the Appendix.

Despite the antecedents, Wilson's 1974 paper ${ }^{22}$ was a watershed for at least three reasons. First, Wilson discussed very clearly how to understand color confinement via the energy stored between a source and sink of color separated by larger and larger distances. Furthermore, he showed that lattice gauge theories confine in the strong-coupling limit (according to his criterion). Second, the spacetime lattice provides a mathematically sound definition of the functional integral. As with canonical quantization, $\frac{46}{4}$ the functional integral for a countable set of degrees of freedom is straightforward to define, and the one for continuously many degrees of freedom is obtained as a limit. Third, Wilson's earlier work on the renormalization group, both in critical phenomena and in the strong interactions, lent credence to the proposal that the lattice formulation of gauge theory could be connected to the gauge-fixed, perturbative formulation of gauge theory. Moreover, all these aspects provided a starting point for others to begin research on lattice gauge theory 
particularly after an influential series of papers $\$ 400$ explained the techniques of statistical mechanics in particle physics language.

Wilson's criterion for color confinement starts with the parallel transporters in Eq. (8). The interacting quark propagater from $x$ to $y$ consists of a properly weighted average of $U_{s}(x, y)$ over all paths $s$. A meson propagator consists of an average of a similar object, $U_{s}(x, y) U_{\bar{s}}(y, x)=U_{s \cup \bar{s}}(x, y)$, where $s(\bar{s})$ is the path taken by the quark (antiquark). Such closed loops of parallel transport are now known as Wilson loops, and even open parallel transporters $U_{s}(x, y)$ of shortest path are often known as Wilson lines. Note that the trace, $\operatorname{tr} U_{\text {loop }}(x, x)$, of a closed loop is gauge invariant.

It is instructive to consider loops for which the spatial coordinates of $x$ and $y$ are the same and set $|x-y|=t$. Furthermore, imagine loops for which $s$ and $\bar{s}$ separate a distance $r$ apart (away from the points where they join). Let us denote such a Wilson loop $U_{r \times t}$, because the shape is more pertinent than the path names $s$ and $\bar{s}$. If the expectation value (when $t \gg r$ )

$$
\left\langle\operatorname{tr} U_{r \times t}\right\rangle \sim e^{-V t}
$$

with $V$ independent of $r$ for large $r$, then quark and antiquark lines can easily separate to large $r$. Such behavior obtains in electrodynamics and signals the absence of confinement. On the other hand, if the expectation value

$$
\left\langle\operatorname{tr} U_{r \times t}\right\rangle \sim e^{-\sigma r t},
$$

then large separations of quark and antiquark are highly improbable. Such behavior is known as the "area law" and corresponds to confinement. ${ }^{22}$

To compute $\left\langle\operatorname{tr} U_{r \times t}\right\rangle$, one employs the functional-integral formulation of field theory. In general, the central objects are correlation functions, so let us introduce several composite, color-singlet fields $\Phi_{i}$, where the subscript labels both spacetime and internal indices, as well as distinguishing one composite field from another. The $n$-point correlation function is given by

$$
\begin{aligned}
\left\langle\Phi_{1} \cdots \Phi_{n}\right\rangle & =\frac{1}{Z} \int \prod_{x, \mu} d U_{x, \mu} \prod_{x} d \psi_{x} d \bar{\psi}_{x} \Phi_{1} \cdots \Phi_{n} e^{-S(U, \psi, \bar{\psi})}, \\
Z & =\int \prod_{x, \mu} d U_{x, \mu} \prod_{x} d \psi_{x} d \bar{\psi}_{x} e^{-S(U, \psi, \bar{\psi})} .
\end{aligned}
$$

Once invariant measures have been specified for the $\mathrm{SU}(N)$ variables ${ }^{51} U_{x, \mu}$ and for the fermion (quark) variables ${ }^{52} \psi_{x}$ and $\bar{\psi}_{x}$, the right-hand sides of these equations are well-defined finite integrals. Mathematicians would say, "they exist." In the limit of infinite temporal extent, such a correlation function yields the vacuumexpectation value of the time-ordered product of the $\Phi_{i} \mathrm{~s}$. Note, however, that lattice gauge theory is well-defined in Euclidean spacetime. The Euclidean signature also leads to minus signs, rather than factors of $i$, in several formulae, but it is not a limitation in principle. 
Equations (12) and (13) specify a canonical average and partition function in classical statistical mechanics. Such systems can exhibit second-order phase transitions, $\frac{53}{5}$ which are characterized by a large correlation length $\xi \gg a$, where $\xi$ measures the falloff of a correlation function, $e^{-|x-y| / \xi}$. Correlation functions with different symmetry-group representations can have different correlation lengths, but all $\xi_{\alpha} \gg a$. At second-order phase transitions, the details of the lattice (e.g., whether the crystal is triclinic, hexagonal, or cubic) become unimportant and the long-distance behavior depends only on the internal symmetries of the interactions. These phenomena translate into particle physics language as follows. One identifies the inverse correlation lengths $\xi_{\alpha}^{-1}$ with particle masses $M_{\alpha}$, because a particle twopoint function behaves like $e^{-M_{\alpha}|x-y|}$. Thus, to define a continuum quantum field theory via lattice field theory, one seeks a point in the space of couplings, such that a hierarchy between the particle masses and the inverse lattice spacing emerges. The hierarchy of scale is the key feature, while Eq. (5) gives the specific example relevant to asymptotically free theories. It means that $M_{\alpha} \propto \Lambda_{F}$, with coefficients that depend on the chromodynamics of quarks and gluons, but not on the lattice.

Because the integrals in Eqs. (12) and (13) exist, they provide a platform for a rigorous construction of quantum field theory $\frac{54}{5}$ To reconstruct a quantummechanical Hilbert space from a Euclidean field theory, the functional integrals must enjoy certain properties, such as a positive action, known as the OsterwalderSchrader axioms 5556 The simplest lattice actions satisfy these conditions. ${ }_{57}^{57}$ That said, the challenge is to lend mathematical rigor to the limiting procedure of the renormalized continuum limit, i.e., one requires a rigorous understanding of critical phenomena. For a historical review of this field, including the role of the renormalization group, see Ref. 58 .

Let us return to Eq. (12) and examine the average of the Wilson loop to learn whether (lattice) gauge theory confines. Taking a hypercubic lattice and a simple gauge-invariant lattice Lagrangian, chosen to reduce to Yang-Mills Lagrangian ${ }^{18 \mid 19}$ in the classical continuum limit, Wilson found

$$
\left\langle\operatorname{tr} U_{m \times n}\right\rangle=\left(2 N / g_{0}^{2}\right)^{m n}=e^{-\ln \left(g_{0}^{2} / 2 N\right) m n} .
$$

for an $m \times n$ rectangular Wilson loop. In fact, the same calculation shows that for any planar Wilson loop, the (dimensionless) area replaces $m n$ in Eq. (14).

The area law follows from a simple property of invariant integration over the gauge group, which states

$$
\int d U U=0
$$

for example, $\int_{-\pi}^{\pi} d \theta e^{i \theta}=0$ for $\mathrm{U}(1)$, and $\int_{-\pi}^{\pi} d \theta \int d^{2} \hat{n} e^{i \hat{\boldsymbol{n}} \cdot \boldsymbol{\sigma} \theta}=0$ for $\mathrm{SU}(2)$. Equation (15) generalizes to say that any color-nonsinglet average over the gauge group vanishes. Color singlets can propagate, while would-be states with color can be considered to have infinite mass. Thus, lattice gauge theory confines. 
Owing to Eq. 15, the area law holds for both abelian as well as nonabelian gauge theories. The salient question, however, is whether the confining behavior persists into the relevant regime of weak coupling. In QED, the long-distance coupling in nature is weak, $\alpha=e^{2} / 4 \pi=1 / 137$, and in QCD weak (bare) coupling corresponds to $a \Lambda_{\mathrm{QCD}} \ll 1$. In fact, the strong-coupling dynamics of (compact) $\mathrm{U}(1)$ lattice gauge theory are influenced by a tangle of magnetic monopoles 5960 unlike what one has in QED. The monopoles provide the crucial insight to prove

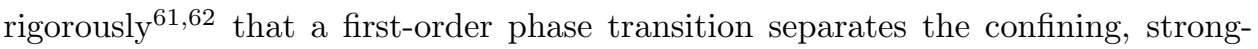
coupling region from a phase with a massless photon and Coulomb interactions. The latter phase is QED, while the confining phase of U(1) lattice gauge theory has nothing to do with QED.

The rigorous proofs fail in the nonabelian case, however. At present, there is no accepted rigorous analytic proof that confinement persists for nonabelian theories into the weak-coupling regime. Several analytical and nonperturbative calculations, taken together, provide strong evidence that the confinement of the strong-coupling limit of lattice gauge theory survives to continuum QCD. First, consider how the exponent in Eq. 11] depends on $g_{0}^{2}$. At strongest coupling, Eq. 14 implies

$$
\sigma a^{2}=\ln \left[g_{0}^{2}(a) / 2 N\right],
$$

whereas at weakest coupling, Eq. (5) requires

$$
\sigma a^{2} \propto e^{-1 / \beta_{0} g_{0}^{2}(a)} .
$$

The issue at hand is whether these two asymptotic behaviors are connected by a smooth function. Michael Creutz's pioneering numerical calculations $\sqrt[63 \mid 64]{6}$ of $\left\langle\operatorname{tr} U_{m \times n}\right\rangle$ and, hence, $\sigma a^{2}$, demonstrated a smooth connection between the two functional forms, with a knee around $g_{0}^{2} \approx 1$. Moreover, Padé extrapolations of high-order strong-coupling expansions anticipate the knee $\frac{65 \mid 66}{6}$ These results thus show no evidence for a first-order transition, so the simplest interpretation is that confinement persists to weak coupling.

The absence of evidence for a phase transition is not the same as evidence for the absence of a phase transition. Indeed, numerical studies $d o$ find first-order transitions in $\mathrm{SU}(N)$ lattice gauge theory $\llbracket 67 / 68$ To do so, one searches in a general space of lattice couplings, including irrelevant couplings. $\mathrm{In} \mathrm{SU}(N)$ in four dimensions, a line of phase transitions ends, and various trajectories in the space of couplings smoothly connect the strongly and weakly coupled regimes. In $\mathrm{U}(1)$, the phase-transition line never ends, so the first-order phase transition cannot be circumvented. It seems unlikely that numerical work has missed a key piece of information about the bulk phase structure of lattice gauge theory. The tool's suitability and the community's expertise seem up to the task.

Adding quarks to lattice gauge theory changes the picture of confinement somewhat. (Lattice-fermion constructions are discussed below.) If the source-sink separation is long enough, it is energetically preferable for a quark-antiquark pair to pop out of the glue and screen the color sink and source. This behavior can be seen 
in a double "high-temperature" series in $1 / g_{0}^{2}$ and $1 / m_{0}$. Terms varying with the area (from the $1 / g_{0}^{2}$ series) and with the perimeter (from the $1 / m_{0}$ series) arise, with the former remaining important for small and intermediate separations, and the latter dominating for large separations.

In addition to the string tension, strong-coupling expansions can be used to compute hadron masses. QCD is expected to have bound states that lie outside the quark model, such as glueballs, which are composed of gluons but no valence quarks. At leading order in strong coupling, various glueballs are degenerate with common mass

$$
M a=4 \ln \left[g_{0}^{2}(a) / 2 N\right] .
$$

The series have been extended through order $g_{0}^{-16}$ for scalar $\left(J^{P C}=0^{++}\right)$, tensor

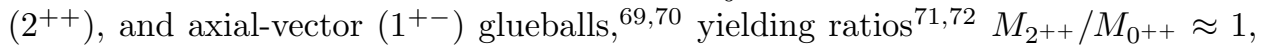
$M_{1^{+-}} / M_{0^{++}}=1.8 \pm 0.3$ (Euclidean spacetime lattice), with similar results from a continuous time Hamiltonian formulation. 73

One can also compute meson and baryon masses. The simplest approach 74 takes both $1 / g_{0}^{2}$ and $1 / m_{0}$ to be small, but the latter would be far from the up and down quarks. Another approach is to exploit mean-field theory techniques from statistical mechanics, which permit the resummation of the $1 / m_{0}$ expansion. This trick amounts to an expansion in $1 / d$, where $d$ is the dimension of spacetime; for $d=4$ the expansion parameter is reasonable small. At strongest coupling, the disorder of the gauge field drives chiral symmetry breaking, 75 and the Goldstone boson (pion) mass satisfies $M_{\pi}^{2} \propto m_{0}\langle\bar{\psi} \psi\rangle$. These calculations also find that nonGoldstone meson masses satisfy $M \propto$ const $+\mathrm{O}\left(m_{0}\right)$ and baryon masses (for $N$ colors) satisfy $M \propto N \times$ const $+\mathrm{O}\left(m_{0}\right)[77 / 78$ We shall return to the implications of spontaneous chiral symmetry breaking at the end of Sect. 3

To end this section, let us discuss the uneasy relationship between fermions and the lattice ${ }^{\mathrm{b}}$ (The level of these paragraphs is somewhat higher, and readers can skip them and proceed to Sect. 3 without much loss.) In the 1974 paper, ${ }^{22}$ Wilson used a lattice fermion Lagrangian with (inverse) free propagator

$$
S^{-1}(p)=i a^{-1} \sum_{\mu=1}^{4} \gamma_{\mu} \sin \left(p_{\mu} a\right)+m_{0},
$$

where each component of the momentum $p$ lies in the interval $(-\pi / a, \pi / a]$. This expression looks like its continuum counterpart not only for $p \sim 0$ but also at the 15 other corners of the Brioullin zone, $p_{\mu} \sim 0 \bmod \pi / a$. In the continuum limit, all 16 modes correspond to physical states, which is known as the "fermion doubling problem." The extra states appear everywhere ${ }^{79}$ For example, they multiply by 16 the fermion-loop contribution to $\beta_{0}$ [the term proportional to $n_{f}$ in Eq. [3] ], and they contribute to the axial anomaly with a pattern of signs $1-4+6-4+1=0$ (in four dimensions). The Lagrangian corresponding to Eq. 19) has an exact chiral

b Whence the remark that Heisenberg and Pauli were prudent not to take up the issue. 
symmetry in the massless limit; hence, the anomaly must vanish in this case (even though this is not desired for QCD).

Several formulations have been introduced to amelioriate the doubling problem. In a Hamiltonian formulation with discrete space and continuous time (and, hence, only 8 states to start with), John Kogut and Leonard Susskind ${ }^{80}$ put the upper two and lower two components of a Dirac spinor on the even and odd sites of the lattice, respectively, reducing the number of degrees of freedom by two. Susskind ${ }^{81}$ later devised a method with one component per site. A Euclidean spacetime lattice version of this method ${ }^{82 / 83}$ is now referred to as staggered fermions. This formulation exactly preserves a subset of chiral symmetry but still has four fermion states for every fermion field. A non-Noether flavor-singlet axial current is anomalous $83[84$

Wilson ${ }^{85}$ introduced a dimension-five term that yields a large mass to the 15 extra states. The axial anomaly is obtained correctly, which is possible because the Wilson term breaks the axial symmetries. In practice, one has a fine-tuning problem here: the mass term and the Wilson term must balance each other to provide the small amount of explicit axial-symmetry breaking of QCD. After this fine-tuning, which can be carried out nonperturbatively, the residual chiral-symmetry breaking is proportional to the lattice spacing. One can add to the action a Pauli term, which is also of dimension five, and then impose Ward identities $87 / 88$ to reduce discretization effects to $\mathrm{O}\left(a^{2}\right)$. For two flavors of Wilson fermions, it is also possible to remove the leading-order discretization effect via an isospin off-diagonal mass term, 8990 which is known as "twisted mass."

On a lattice, chiral symmetry and the doubling problem are deeply connected, which is encapsulated in the Nielsen-Ninomiya theorem 91921 A way around this theorem comes from the Ginsparg-Wilson relation, 93 which uses renormalizationgroup ideas to derive a minimal condition on lattice chiral symmetry (for Dirac fermions). The Ginsparg-Wilson relation reads

$$
\gamma_{5} D+D \gamma_{5}=a D \gamma_{5} D
$$

where $D$ is the lattice Dirac operator. The Nielsen-Ninomiya theorem assumes the right-hand side vanishes and, thus, does not apply. Solutions to the GinspargWilson relation ${ }^{94}$ are compatible with a suitably modified chiral transformation ${ }^{97}$ but are computationally more demanding than the other methods. This setup allows

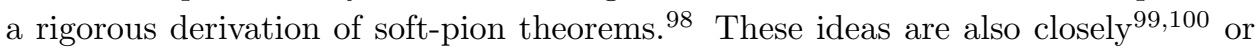
intimately $101 / 102$ related to ideas to formulate chiral gauge theories (such as the standard electroweak interaction) on the lattice.

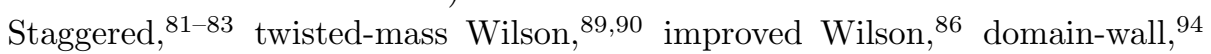
and overlap ${ }^{95}$ fermions are all used in the large-scale computations discussed below.

\section{The Origin of (Your) Mass}

Although strong-coupling expansions provided new insight into gauge theories, it became clear that they would not offer a path to small, robust error bars. Today, a 
set of numerical Monte Carlo techniques are the largest focus of research in lattice gauge theory. In many cases, for example, the computation of hadronic matrix elements in electroweak processes, the goal is to provides a solid number with a full error budget. To understand mass, however, one would like to have more than numbers, but also a qualitative understanding. As we shall see, numerical calculations have played a key role here too.

Let us begin with a short explanation of the numerical methods. In all cases of interest, the action in Eq. 12 can be written $S=S_{\text {gauge }}+\bar{\psi}(D+m) \psi$, where $D$ is a matrix with spacetime, color, flavor, and Dirac indices, and $m$ is a mass matrix (diagonal in all indices). To obtain a nonzero result, the number of fermion and antifermion fields in Eq. (12) must be the same. Suppose the number is $A$; that means that the product of $\Phi$ s can be re-expressed as

$$
\prod_{i=1}^{n} \Phi_{i}=\phi(U) \prod_{a=1}^{A} \bar{f}_{a}(U) \psi \bar{\psi} f_{a}(U)
$$

where $\bar{f}_{a}(U)$ and $f_{a}(U)$ account for all structure attached to fermions and antifermions on the left-hand side, and $\phi(U)$ stands for whatever remains. To calculate hadron masses, we need two-point functions ( $n=2$ on the left-hand side) for mesons ( $A=2$ on the right-hand side) and baryons $(A=3)$. These two-point functions can be expressed as $\left(x_{4}>0\right)$

$$
\left\langle\Phi_{i}^{\dagger}\left(x_{4}\right) \Phi_{j}(0)\right\rangle=\sum_{r=0}^{\infty}\left\langle 0\left|\hat{\Phi}_{i}^{\dagger}\right| r\right\rangle\left\langle r\left|\hat{\Phi}_{j}\right| 0\right\rangle \exp \left(-M_{r} x_{4}\right)
$$

where the $\Phi_{i}$ have specific three-momentum and flavor quantum numbers, and $M_{r}$ is the energy of the $r$ th radial excitation with the quantum numbers of $\Phi_{i}$. For three-momentum $\boldsymbol{p}=\mathbf{0}$, energy means mass. For simple actions, Eq. (22) is a

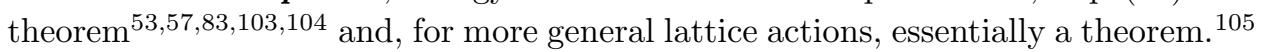
Given the left-hand side from a numerical computation, the masses are extracted by fitting the numerical data to the right-hand side. These fits can be improved by choosing $x_{4}$ large enough to suppress highly excited states and by choosing the $\Phi_{i}$ to project mostly onto one specific state.

The integration over fermionic variables can be carried out by hand, yielding

$$
\begin{array}{r}
\left\langle\phi(U) \prod_{a=1}^{A} \bar{f}_{a} \psi \bar{\psi} f_{a}\right\rangle=\frac{1}{Z} \int \prod_{x, \mu} d U_{x, \mu} \phi(U) \operatorname{det}_{a, b}\left\{\bar{f}_{a}[D(U)+m]^{-1} f_{b}\right\} \times \\
\operatorname{Det}[D(U)+m] e^{-S_{\text {gauge }}(U)},
\end{array}
$$

where $\operatorname{det}_{a, b}$ is a normal determinant over the enumeration of fermion fields, while Det denotes a determinant over spacetime, color, flavor, and Dirac indices. Physically, $\operatorname{det}_{a, b} \bar{f}_{a}[D(U)+m]^{-1} f_{b}$ represents the propagators of valence quarks in the $n$-point function, while $\operatorname{Det}[D(U)+m]$ denotes sea quarks - virtual quark-antiquark pairs bubbling out of the stew of gluons. 
The number of independent variables of integration is huge if the spatial extent is to be larger than a hadron and the lattice spacing much smaller than a hadron. The only feasible numerical technique for computing such integrals is a Monte Carlo method with importance sampling. That means to generate $C$ configurations of $\left\{U_{x, \mu}, \forall x, \mu\right\}^{(c)}$ chosen randomly with weight $\operatorname{Det}[D(U)+m] e^{-S_{\text {gauge }}(U)}$. Then

$$
\left\langle\phi(U) \prod_{a} \bar{f}_{a} \psi \bar{\psi} f_{a}\right\rangle=\lim _{C \rightarrow \infty} \frac{1}{C} \sum_{c=1}^{C} \phi\left(U^{(c)}\right) \operatorname{det}_{a, b}\left\{\bar{f}_{a}\left[D\left(U^{(c)}\right)+m\right]^{-1} f_{b}\right\} .
$$

In practice, $C$ is finite but as large as possible. The details of the numerical algorithms lies beyond the scope of this article; for a pedagogical review, see Ref. 106.

The second-most computationally demanding part of this procedure is to obtain the valence-quark propagators $\bar{f}_{a}[D(U)+m]^{-1} f_{b}$. The most demanding part is to account for the sea-quark factor $\operatorname{Det}[D(U)+m]$ in the importance sampling. Early mass calculations thus used a valence approximation, 107 computing each $\bar{f}_{a}\left[D\left(U^{(c)}\right)+m\right]^{-1} f_{b}$ while replacing $\operatorname{Det}\left[D\left(U^{(c)}\right)+m\right]$ with 1 . In addition, the elimination of bare parameters in favor of physical quantities absorbs an implicitly specified part of the physical effects of the sea quarks. The valence approximation is better known as the quenched approximation, from an analogy with condensedmatter systems! 108

There are too many quenched calculations of hadron masses in the literature to provide a useful survey. Nowadays, the inclusion of sea quarks is commonplace. Indeed, in some important applications, such as the thermodynamics of QCD, the sea quarks play an absolutely crucial role. The remainder of this article focuses, therefore, on numerical calculations that include the sea of up, down, and strange quarks; these are usually called $2+1$-flavor calculations. In some cases, charmed sea

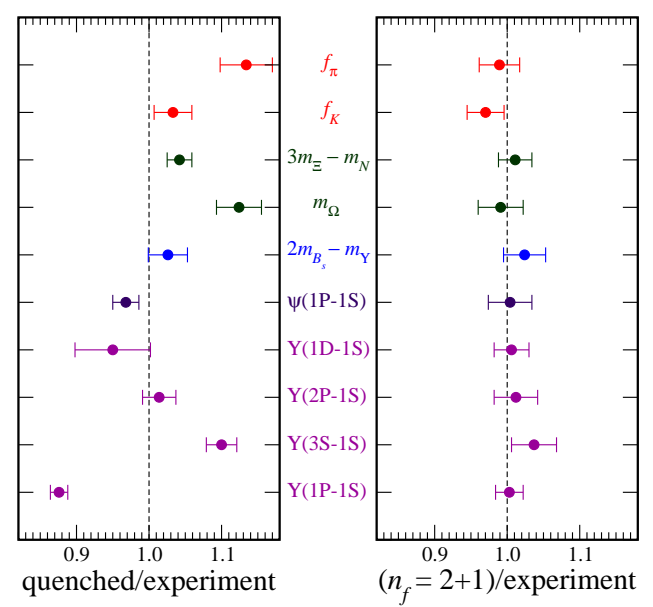

Fig. 1. Comparison of quenched and $2+1$ lattice-QCD calculations, showing only statistical errors (to assess the systematics of quenching) $\frac{109}{109}$ Free parameters $\left(g_{0}^{2}(a), \frac{1}{2}\left(m_{u}+m_{d}\right), m_{s}\right)$ fixed with the 2S-1S splitting of bottomonium, $M_{\pi}$, and $M_{K}$. 
quarks are included as well, and these are known as $2+1+1$-flavor calculations.

Figure 1 shows a comparison of quenched and $2+1$ calculations for a wide variety of masses and pseudoscalar-meson decay constants. 109 The results of the latticeQCD calculations are divided by their corresponding entries in the (2002 edition of the) review of particle physics from the Particle Data Group (PDG) ${ }^{110}$ Results should ideally lie close to 1 . The quenched results lie with $10-15 \%$ of PDG values, sometimes closer, but the pattern of (nonstatistical) variation is hard to understand. Upon adding $2+1$ flavors of sea quarks, the discrepancies disappear.

Computational science often develops in a way that festoons the basics with many specialized methodological improvements. Nonexperts often react by putting the whole process into a black box to shield themselves from the details. They are then comforted by genuine predictions: calculations for which the correct result was not known in advance, but which are then confirmed by other means, e.g., experimental measurements.

Soon after the publication of in Fig. 1, lattice QCD enjoyed several such predictions, including the shape of form factors in semileptonic $D$ decays, ${ }^{111}$ the mass of the $B_{c}$ meson (composed of a bottom quark and a charmed antiquark), ${ }^{112}$ the decay constants of charmed mesons, $\frac{113}{113}$ and the mass of the $\eta_{b}$ meson (the lightest bottomonium state) ${ }^{114}$ Figure 2 shows measurements of the form factors for $D \rightarrow K \ell \nu$ and $D \rightarrow \pi \ell \nu$ by the CLEO experiment ${ }^{115}$ overlaying a lattice-QCD calculation ${ }^{111}$ with an improved visualization of systematic errors. ${ }^{116}$ The CLEO data ${ }^{115}$ are the most precise among several experiments confirming the lattice-QCD calculation;

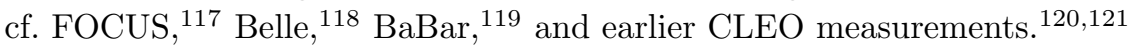

Before turning to hadron-mass calculations, let us take stock of the numerical results discussed so far. Section 2 noted that Monte Carlo calculations of simple quantities such as Wilson loops agreed with both strong-coupling and weak-coupling expansions, in their respective domains of applicability. Here, we have seen that quarkonium masses and some other properties of heavy-quark systems-as well as leptonic decay constans $f_{\pi}$ and $f_{K}$-agree very well with experimental measure-
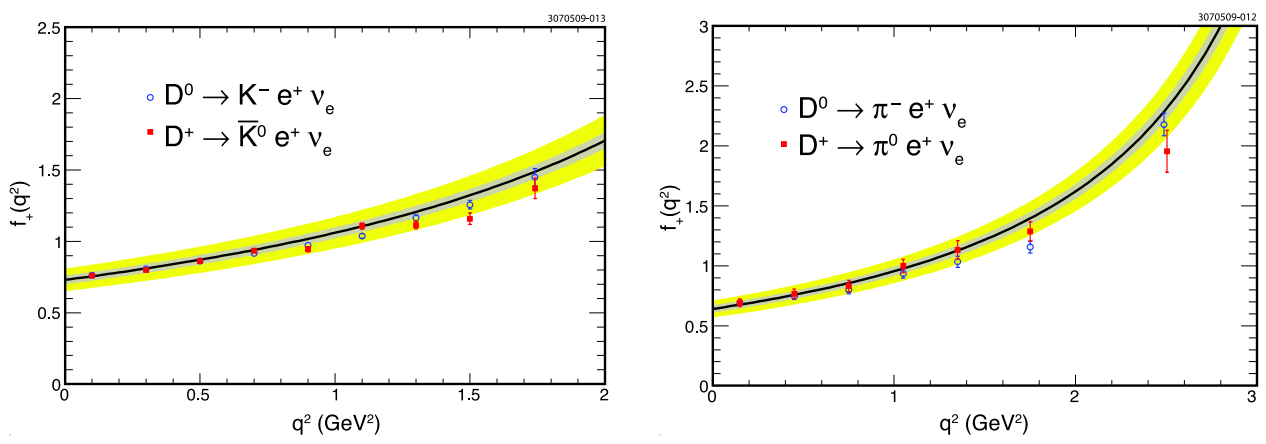

Fig. 2. Comparisor $[115$ of $2+1$ lattice-QCD calculations of $D$-meson form factors $\$ 111116$ (curves with error bands) with measurements from CLEO 115 (points with error bars). 
ments, even when those were not known ahead of time. When combined with the numerous self-consistency checks that every modern large-scale lattice-QCD calculation undergoes, it is fair to say that the techniques for generating and analyzing numerical data have matured. In particular, the standards for estimating full error budgets have become, by and large, high.

With confidence bolstered by these remarks, let us now examine recent calculations of the hadron mass spectrum. A summary is shown in Fig. 3. More details about the underlying work can be found in the review from which this plot is taken $\frac{122}{}$ or in a comprehensive review of hadron mass calculations. 134 The most important features are as follows. Many different groups of researchers (symbol shape and color) have carried out these calculations, and they all find broad agreement with nature. They use different fermion formulations (symbol shape) and a different range of lattice spacing and quark masses (symbol color). The total errors in many cases are small. In particular, the nucleon mass - the main contributor to everyday mass - has an error of around $2 \%$.

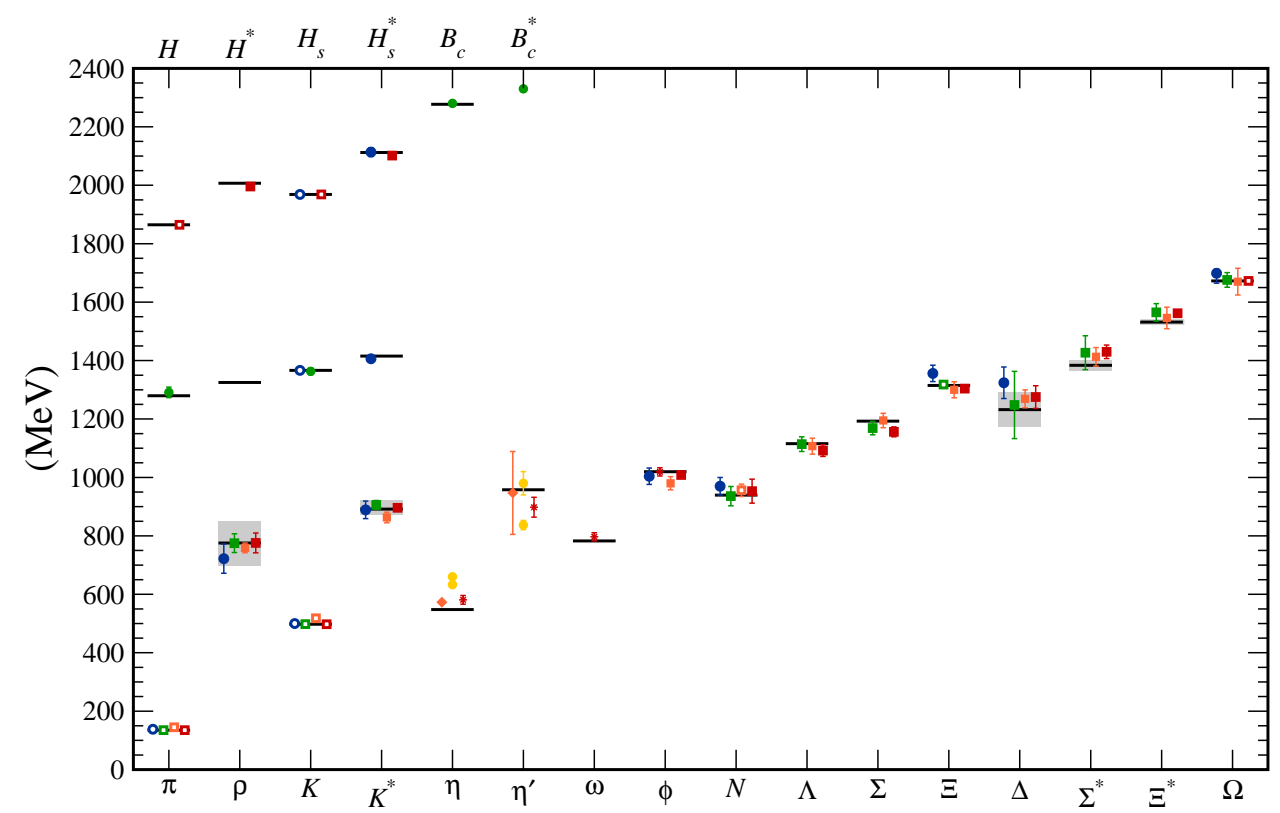

Fig. 3. Hadron mass spectrum with $2+1$ flavors of sea quarks, from Ref. 122 Results for many light mesons and baryons are from MILC $123 \mid 124$ PACS-CS, 125 BMW 126 and QCDSF $\stackrel{127}{127}$ Results for the $\eta$ and $\eta^{\prime}$ mesons are from RBC \& UKQCD, 128 Hadron Spectrum, 129 and UKQCD 130 Result for the $\omega$ meson is from Hadron Spectrum!129 Results for heavy-light mesons are from Fermilab-MILC, ${ }^{131}$ HPQCD, 132$]$ and Mohler \& Woloshyn!133 $b$-flavored meson masses are offset by $-4000 \mathrm{MeV}$. Circles, squares, and diamonds denote staggered, Wilson, and chiral sea quarks, respectively. Asterisks represent anisotropic lattices, $a_{4} / a_{i}<1$. Open symbols denote inputs; filled symbols and asterisks denote output results. Red, orange, yellow, green, and blue denote increasing numbers of ensembles (i.e., range of lattice spacing and depth of sea quark masse). Horizontal bars (gray boxes) denote experimentally measured masses (widths). 
Figure 3 shows only the lowest-lying state in each channel, cf. Eq. 222. Excited states pose more technical challenges, starting with a lower signal-to-noise ratio in the Monte Carlo estimates of the two-point functions. Nevertheless, recent progress in this area has been encouraging. An example for mesons is shown in Fig. 4 . A further noteworthy feature of these calculations is that the same techniques that disentangle the excited-state spectrum also yield mixing angles. These mixing angles agree well with corresponding experimental measurements. ${ }^{135}$ Figure 4 also shows results for exotic mesons, which have $J^{P C}$ quantum numbers that are inaccessible from the quark model, and for glueballs (in the quenched approximation, 136 though more recent glueball calculations with $2+1$ sea quarks find similar glueball masses 137 ). These glueball masses validate the axial-vector/scalar but not the tensor/scalar ratios of strong coupling. Excited baryon mass calculations have also been carried out $138 \mid 139$ and will be tested by experiments at Jefferson Laboratory! ${ }^{140}$

Figure 3 shows that we have obtained a solid, quantitative understanding of the mass of simple hadrons, and Fig. 4 shows that this understanding is improving for more complicated hadrons. To understand how the mass is generated, however, a qualitative understanding is also necessary. Here, let us return to the valence approximation to obtain a physical picture.

In electrodynamics, one measures the force by varying the distance between two static charges. The force arises from the energy stored in the electric dipole field between the two charges. As a quantum system, the field actually has discrete energy levels, the lowest being the (semiclassical) potential energy (whose gradient yields the force). The same holds for the chromoelectric dipole field between a

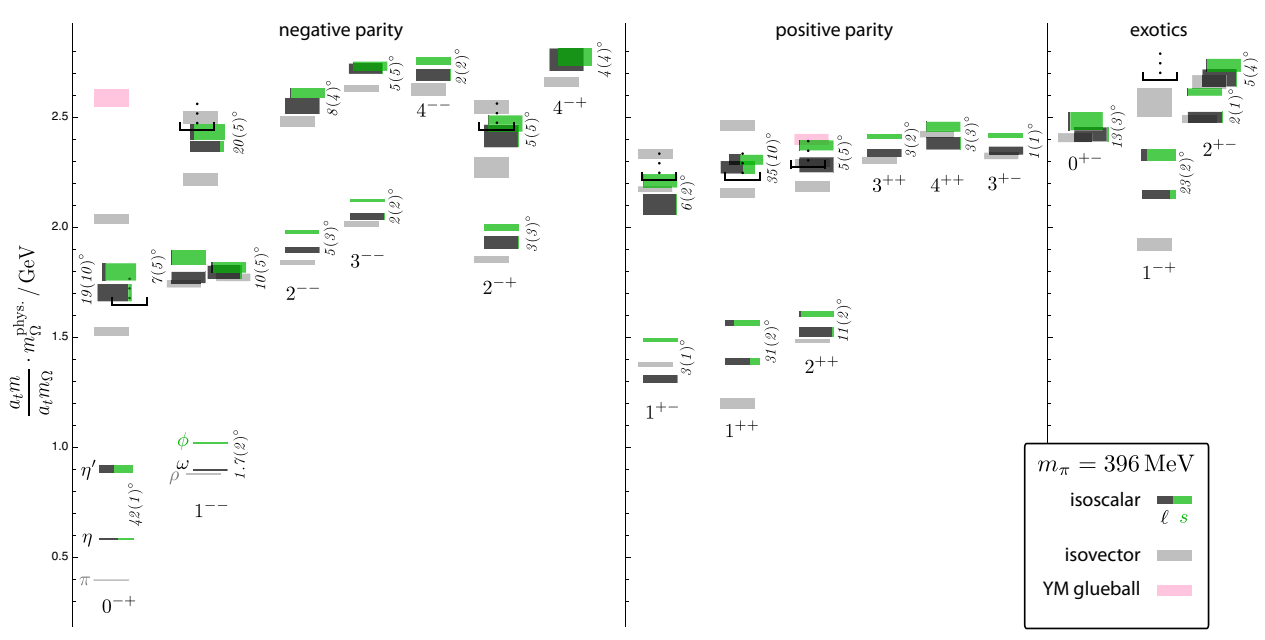

Fig. 4. Excited-state meson spectrum, 129 including isoscalar- $\bar{s} s$ mixing (shades of green) and pure-gauge glueballs (pink). 
static source and static sink of color. Now, however, the shape of the dipole field is influenced by the gluon self-interaction: chromoelectric field lines attract each other.

Figure 5 shows the excitation spectrum of the chromoelectric dipole field $\frac{141}{1}$ At short distances, the level spacing and ordering is in accord with asymptotic freedom. For example, the lowest level, $V(r)$ is Coulombic up to logarithmic corrections. As $r$ increases, the spacing between the levels changes. At a separation of around $2 \mathrm{fm}$, the level ordering rearranges to that of a string, but the level spacing does not become fully string-like until larger separations. ${ }^{142}$ At large distances, the potential $V(r)$ becomes linear in $r$; this behavior is the area law of Eq. 111), now, however, near the continuum limit and only at large enough distances. A vivid picture of the flux tube has it narrowing as $r$ increases, owing to the attraction between gluons, but the details suggest that the flux tube retains diameter a bit less than $1 \mathrm{fm} \cdot 143$ At hadronic length scales, it looks more like a sausage than a string. This picture holds even when quarks are added to the calculations: the linear region persists out to around $1.25 \mathrm{fm}\left(r \sigma^{1 / 2} \approx 2.5\right)$, at which point the flux tube breaks ${ }^{144}$

The linear rise of the potential for $r \gtrsim 0.4 \mathrm{fm}$ provides a striking explanation of the hadron masses $\frac{145}{145}$ The energy stored in a flux-tube of length $L$ and string tension $\sigma$ is simply $\sigma L$. (Here, $L$ should be large enough to be in the linear regime but shorter that the string-breaking distance.) Imagine attaching a massless quark

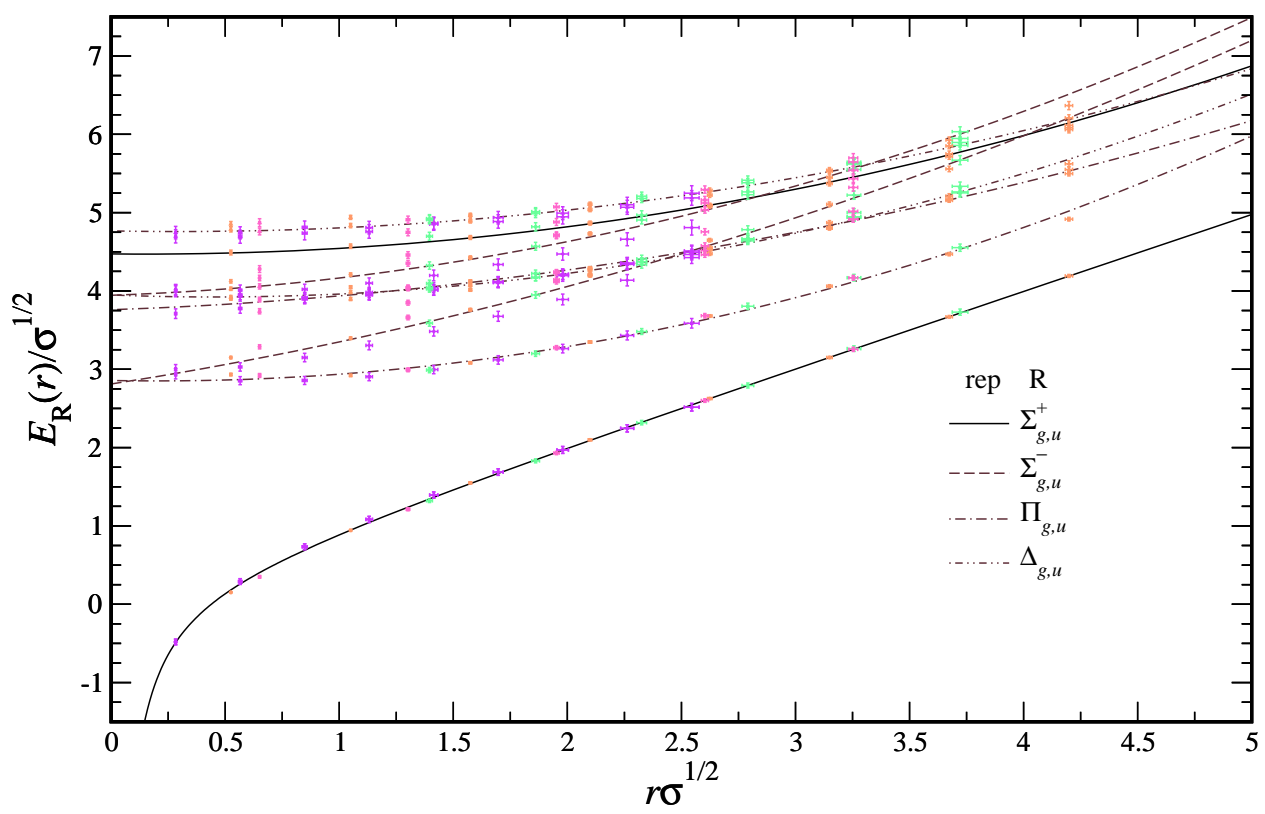

Fig. 5. Excited-state spectrum of potentials 141 The representations $R$ correspond to the gluonic angular momentum along the source-sink axis, with subscript $g(u)$ for $C P= \pm 1$, and for $\Sigma$ wave a superscript for parity upon reflection through the midpoint. To convert from string-tension units to physical units, note $\sigma^{1 / 2} \approx 420 \mathrm{MeV} \approx 2.1 \mathrm{fm}^{-1}$. Plot from Ref. 122 
to one end of the tube and an antiquark to the other. The ends must move with the speed of light, and the total mass $M=E / c^{2}$ of this "meson" is

$$
M=\int_{-L / 2}^{L / 2} \frac{\sigma d x}{\left[1-v(x)^{2}\right]^{1 / 2}}=\int_{-L / 2}^{L / 2} \frac{\sigma d x}{\left[1-(2 x / L)^{2}\right]^{1 / 2}}=\frac{1}{2} \pi \sigma L,
$$

where the denominator accounts for relativistic motion. The kinetic energy increases the mass by $\frac{\pi}{2}-1 \approx 60 \%$. The angular momentum of this system is

$$
J=\int_{-L / 2}^{L / 2} \frac{\sigma v(x) x d x}{\left[1-v(x)^{2}\right]^{1 / 2}}=\frac{2}{L} \int_{-L / 2}^{L / 2} \frac{\sigma x^{2} d x}{\left[1-(2 x / L)^{2}\right]^{1 / 2}}=\frac{1}{8} \pi \sigma L^{2}=\frac{M^{2}}{2 \pi \sigma},
$$

where the last step comes from eliminating $L$ in favor of $M$. Experimental measurements of meson masses and spin satisfy such linear relationships - known as Regge trajectories-between $J$ and $M^{2}$, albeit with nonzero intercepts (from effects neglected here). Equations (25) and 26) are interesting because they are simple. The idea behind them is supported, however, by the empirical observation that heavylight meson wave functions computed directly with lattice gauge theory coincide with Schrödinger wave functions determined from a relativistic kinetic energy and the heavy-quark potential computed with lattice gauge theory 146

Let us return to chiral symmetry breaking. Before quarks or partons had been proposed, Nambu ${ }^{147}$ pointed out that the small mass of the pion $(140 \mathrm{MeV})$ could be explained if an axial symmetry was spontaneously broken. QCD possesses such symmetries in the limit of vanishing quark mass. Indeed, in this idealization, the pion mass would vanish by the following theorem 148

$$
M_{\pi}^{2}\langle\bar{\psi} \psi\rangle=0
$$

This picture has been demonstrated via quantiative lattice-QCD calculations of the chiral condensate $\frac{149}{149}$ firmly establishing $\langle\bar{\psi} \psi\rangle \neq 0$. The nonzero pion mass arises owing to the explicit symmetry breaking from the up and down quark masses. As a consequence, one expects $M_{\pi}^{2} \propto m_{q}$, which has been amply demonstrated in lattice QCD $\sqrt{124134}$ Since the nucleon and pion experience residual strong interactions, the nucleon is surrounded with a cloud of pions. The size of the nucleon, and other hadrons is, thus, dictated by the pion Compton wavelength; the density of nuclear material is proportional to $M_{N} M_{\pi}^{3}$.

The richness of everyday life stems from chemistry, which, in turn, hinges both on an attractive force between protons and neutrons to hold atomic nuclei together, and a short-range repulsive force to aid nuclear stability. In QCD, the attractive force is akin to van der Waals forces among molecules and can be vividly and successfully modeled by meson exchange, particularly pion exchange. The detailed, first-principles study of these forces is just beginning 150|151 Recent developments have been encouraging and illustrate that the origin of mass is not the only exciting problem in physics. 


\section{Summary and Outlook}

The origin of mass is a compelling problem with many facets. This article has touched on only one, the origin of mass of everyday objects, which can be pinpointed directly to the protons and neutrons in atomic nuclei. Remarkably, most of the nucleon mass has a dynamical origin: strong confining forces influenced by chiral symmetry breaking generate the mass and size of nucleons and, hence, nuclei. We understand these dynamics as quantum chromodynamics. With powerful numerical calculations based on lattice gauge theory, we have disentangled puzzles and verified many conjectures.

Because of asymptotic freedom, QCD as a quantum field theory holds consistently at all energy scales. That said, as the exploration of particles physics unfolds in the future, it is conceivable that physicists will discover a substructure to quarks or a unification of the chromodynamic interaction with the other gauge interactions of the standard model. Such discoveries would relegate the SU(3) gauge symmetry of QCD to a (relatively) low-energy description of nature. Moreover, in such frameworks a high-energy value of the QCD gauge coupling is specified, and, in many cases, a set of thresholds affecting its running is specified as well. One can thus imagine connecting $\Lambda_{\mathrm{QCD}}$ to the scales of a more fundamental, more microscopic theory of (most) everything. Even so, one would still have to concede that chromodynamics generate everyday mass. The key physics is the attraction of gluons to each other, the relativistic kinetic energy of light quarks, and the constraints imposed by dynamical chiral symmetry breaking.

\section{Acknowledgments}

My sense of the history of lattice gauge theory has been shaped by conversations over the years with Bernd Berg, Robert Finkelstein, Chris Hill, Peter Lepage, Paul Mackenzie, Gernot Münster, Don Petcher, Junko Shigemitsu, Don Weingarten, and Ken Wilson, among others. In particular, Gernot Münster told me about Werner Heisenberg and Piet Hein.

Fermilab is operated by Fermi Research Alliance, LLC, under Contract No. DEAC02-07CH11359 with the United States Department of Energy. I thank the Galileo Galilei Institute for Theoretical Physics for hospitality, and the INFN for partial support, while this article was being completed.

\section{Appendix: Heisenberg's Gitterwelt and Hein's Soma}

Werner Heisenberg hoped for more from the lattice than mere mathematical rigor. In a 1930 letter to Niels Bohr, he argued that a universe with a fundamental length, such as a spatial lattice spacing, would not suffer from many problems (then) facing quantum field theory and nuclear and atomic physics. For a translation of the letter and reconstruction of Heisenberg's ideas, see Carazza and Kragh ${ }^{152}$ Bohr 
responded disapprovingly to the idea. Heisenberg did not publish a paper on his "Gitterwelt" ("lattice world"), as it came to be known, although he did make a technical remark that the lattice tames the ultraviolet divergence in the electron's self energy ${ }^{153}$ Nevertheless, Heisenberg's Gitterwelt developed a philosophical and scientific following, which was met with some disdain $\sqrt{152 \mid 154}$ I've been told ${ }^{\sqrt{155}}$ that when Wilson presented his lattice gauge theory in a seminar at Caltech, he deflected an aggressive line of questioning from Richard Feynman with, "I am not a kook; this is not a kook's lattice!" This give-and-take seems to reflect a lingering apprehension against a lattice as fundamental, while underappreciating its mathematical utility.

A lasting outgrowth of Heisenberg's lattice world lies not in theoretical physics but in a geometric puzzle called Soma, which was created by the Danish inventor and poet Piet Hein $156 \mid 157$ Sometime in the early 1930s, Hein - among other avocations a physics groupie - attended a lecture by Heisenberg in Copenhagen. Whether the lecture was on the quantization of QED or on the lattice world, no one seems to know. Bored, Hein sketched a small three-dimensional lattice on a piece of paper and realized something interesting. The seven irregular shapes made from three or four cubes (see Fig. 6) can be assembled into a larger $3 \times 3 \times 3$ cube. These pieces can be assembled in many other mind-bending ways, and Soma has become one of the most popular three-dimensional puzzles of all time.

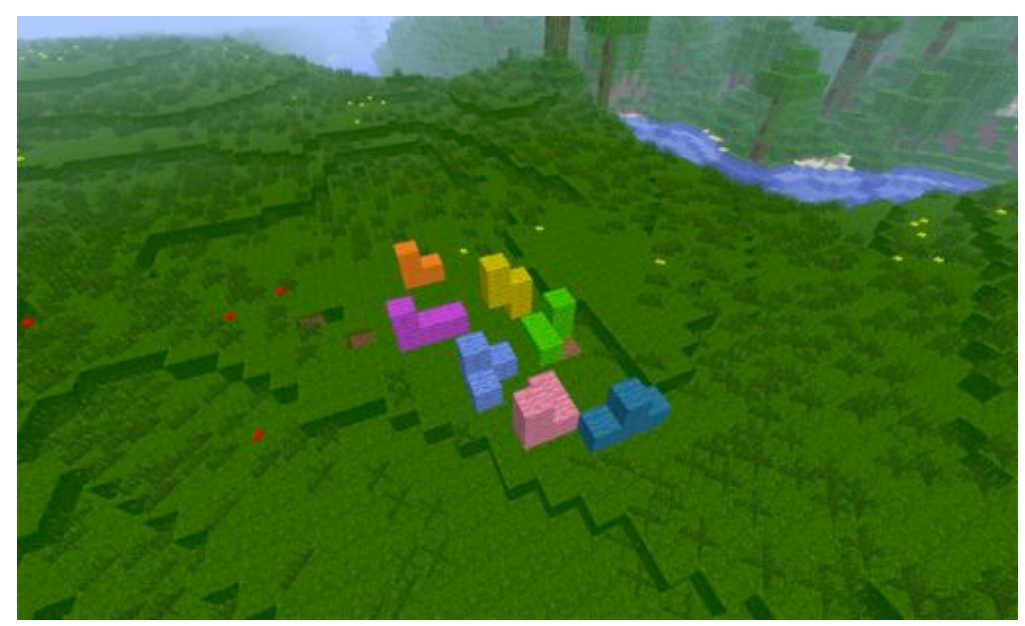

Fig. 6. The seven shapes in Soma grew out of lattice field theory. Graphic by Alexander Kronfeld created with Minecraft (C) 2009-2012 Mojang).

\section{References}

1. G. Aad et al., Observation of a new particle in the search for the standard model Higgs boson with the ATLAS detector at the LHC, Phys. Lett. B716, 1-29, (2012). doi: $10.1016 /$ j.physletb.2012.08.020. 
2. S. Chatrchyan et al., Observation of a new boson at a mass of $125 \mathrm{GeV}$ with the CMS experiment at the LHC, Phys. Lett. B716, 30-61, (2012). doi: 10.1016/j.physletb. 2012.08.021.

3. T. Aaltonen et al., Evidence for a particle produced in association with weak bosons and decaying to a bottom-antibottom quark pair in Higgs boson searches at the Tevatron, Phys. Rev. Lett. 109, 071804, (2012). doi: 10.1103/PhysRevLett.109.071804.

4. J. J. Thomson, On the structure of the atom, Phil. Mag. 7, 237-265, (1904). doi: $10.1080 / 14786441308635024$.

5. H. Nagaoka, Kinetics of a system of particles illustrating the line and the band spectrum and the phenomena of radioactivity, Phil. Mag. 7, 445-455, (1904). doi: 10.1080/14786440409463141.

6. H. Geiger and E. Marsden, On a diffuse reflection of the $\alpha$-particles, Proc. Roy. Soc. Lond. A. 82, 495-500, (1909). doi: 10.1098/rspa.1909.0054.

7. E. Rutherford, The scattering of $\alpha$ and $\beta$ particles by matter and the structure of the atom, Phil. Mag. 21, 669-688, (1911). doi: 10.1080/14786440508637080.

8. J. Chadwick, Possible existence of a neutron, Nature. 129, 312, (1932). doi: 10.1038/ $129312 \mathrm{a} 0$.

9. R. P. Feynman, Very high-energy collisions of hadrons, Phys. Rev. Lett. 23, 14151417, (1969). doi: 10.1103/PhysRevLett.23.1415.

10. R. P. Feynman, Photon-Hadron Interactions. (Benjamin, Reading, MA, 1972).

11. G. Zweig. An SU(3) model for strong interaction symmetry and its breaking. URL http://cdsweb.cern.ch/search.py?recid=352337 (1964).

12. G. Zweig. An SU(3) model for strong interaction symmetry and its breaking II. URL http://cdsweb.cern.ch/search.py?recid=570209 (1964).

13. M. Gell-Mann, A schematic model of baryons and mesons, Phys. Lett. 8, 214-215, (1964). doi: 10.1016/S0031-9163(64)92001-3.

14. O. W. Greenberg, Spin and unitary spin independence in a paraquark model of baryons and mesons, Phys. Rev. Lett. 13, 598-602, (1964). doi: 10.1103/ PhysRevLett.13.598.

15. M.-Y. Han and Y. Nambu, Three triplet model with double SU(3) symmetry, Phys. Rev. 139, B1006-B1010, (1965). doi: 10.1103/PhysRev.139.B1006.

16. J. D. Bjorken and E. A. Paschos, Inelastic electron-proton and $\gamma$-proton scattering and the structure of the nucleon, Phys. Rev. 185, 1975-1982, (1969). doi: 10.1103/ PhysRev.185.1975.

17. H. Fritzsch, M. Gell-Mann, and H. Leutwyler, Advantages of the color-octet gluon picture, Phys. Lett. B47, 365-368, (1973). doi: 10.1016/0370-2693(73)90625-4.

18. C. N. Yang and R. L. Mills, Conservation of isotopic spin and isotopic gauge invariance, Phys. Rev. 96, 191-195, (1954). doi: 10.1103/PhysRev.96.191.

19. R. Shaw. The problem of particle types and other contributions to the theory of elementary particles. PhD thesis, Cambridge University, (1955).

20. H. D. Politzer, Reliable perturbative results for strong interactions, Phys. Rev. Lett. 30, 1346-1349, (1973). doi: 10.1103/PhysRevLett.30.1346.

21. D. J. Gross and F. Wilczek, Ultraviolet behavior of nonabelian gauge theories, Phys. Rev. Lett. 30, 1343-1346, (1973). doi: 10.1103/PhysRevLett.30.1343.

22. K. G. Wilson, Confinement of quarks, Phys. Rev. D10, 2445-2459, (1974). doi: 10.1103/PhysRevD.10.2445.

23. M. Gell-Mann and F. E. Low, Quantum electrodynamics at small distances, Phys. Rev. 95, 1300-1312, (1954). doi: 10.1103/PhysRev.95.1300.

24. K. G. Wilson, Non-Lagrangian models of current algebra, Phys. Rev. 179, 1499-1512, (1969). doi: 10.1103/PhysRev.179.1499. 
25. K. G. Wilson, The renormalization group and strong interactions, Phys. Rev. D3, 1818-1846, (1971). doi: 10.1103/PhysRevD.3.1818.

26. W. Celmaster and R. J. Gonsalves, The renormalization prescription dependence of the QCD coupling constant, Phys. Rev. D20, 1420, (1979). doi: 10.1103/PhysRevD. 20.1420 .

27. R. K. Ellis, W. J. Stirling, and B. R. Webber, QCD and Collider Physics. (Cambridge University, Cambridge, U.K., 1996).

28. M. L. Perl, E. R. Lee, and D. Loomba, Searches for fractionally charged particles, Annu. Rev. Nucl. Part. Sci. 59, 47-65, (2009). doi: 10.1146/ annurev-nucl-121908-122035.

29. K. G. Wilson, The origins of lattice gauge theory, Nucl. Phys. Proc. Suppl. 140, 3-19, (2005). doi: 10.1016/j.nuclphysbps.2004.11.271.

30. G. 't Hooft, Renormalization of massless Yang-Mills fields, Nucl. Phys. B33, 173199, (1971). doi: 10.1016/0550-3213(71)90395-6.

31. G. 't Hooft and M. J. G. Veltman, Regularization and renormalization of gauge fields, Nucl. Phys. B44, 189-213, (1972). doi: 10.1016/0550-3213(72)90279-9.

32. K. G. Wilson, Renormalization group and critical phenomena I: Renormalization group and the Kadanoff scaling picture, Phys. Rev. B4, 3174-3183, (1971). doi: 10.1103/PhysRevB.4.3174.

33. K. G. Wilson, Renormalization group and critical phenomena II: Phase space cell analysis of critical behavior, Phys. Rev. B4, 3184-3205, (1971). doi: 10.1103/ PhysRevB.4.3184.

34. K. G. Wilson, The renormalization group: Critical phenomena and the Kondo problem, Rev. Mod. Phys. 47, 773-840, (1975). doi: 10.1103/RevModPhys.47.773.

35. K. G. Wilson, The renormalization group and critical phenomena, Rev. Mod. Phys. 55, 583-600, (1983). doi: 10.1103/RevModPhys.55.583.

36. N. H. Christ, R. Friedberg, and T. D. Lee, Gauge theory on a random lattice, Nucl. Phys. B210, 310-336, (1982). doi: 10.1016/0550-3213(82)90123-7.

37. G. Wentzel, Zum Problem des statischen Mesonfeldes, Helv. Phys. Acta. 13, 269-308, (1940). doi: 10.5169/seals-111066. (E) ibid. 14, 633 (1941).

38. L. I. Schiff, Lattice-space quantization of a nonlinear field theory, Phys. Rev. 92, 766-779, (1953). doi: 10.1103/PhysRev.92.766.

39. K. G. Wilson, Future directions in particle theory, In eds. D. G. Cassel and D. L. Kreinick, Lepton-Photon Symposium 1983, pp. 812-834. Newman Laboratory, Cornell University, Ithaca, NY, (1983).

40. F. J. Wegner, Duality in generalized Ising models and phase transitions without local order parameters, J. Math. Phys. 12, 2259-2272, (1971). doi: 10.1063/1.1665530.

41. J. Smit. Unpublished. Noted in Refs. 39 and 42 . (1972).

42. J. Smit, Introduction to quantum fields on a lattice: A robust mate. (Cambridge University, Cambridge, UK, 2002). Endnote to Chapter 4 on p. 257.

43. A. M. Polyakov, Compact gauge fields and the infrared catastrophe, Phys. Lett. B59, 82-84, (1975). doi: 10.1016/0370-2693(75)90162-8. This publication builds on unpublished work on gauge fields on a lattice.

44. G. Wentzel, Quantum Theory of Fields. (Interscience, New York, 1949).

45. L. I. Schiff, Quantum Mechanics. (McGraw-Hill, New York, 1968).

46. W. Heisenberg and W. Pauli, Quantendynamik der Wellenfelder, Z. Phys. 56, 1-61, (1929). doi: 10.1007/BF01340129.

47. C. Rebbi, Lattice Gauge Theories and Monte Carlo Simulations. (World Scientific, Singapore, 1983). A reprint volume.

48. R. Balian, J. M. Drouffe, and C. Itzykson, Gauge fields on a lattice 1: General 
outlook, Phys. Rev. D10, 3376-3395, (1974). doi: 10.1103/PhysRevD.10.3376.

49. R. Balian, J. M. Drouffe, and C. Itzykson, Gauge fields on a lattice 2: Gauge invariant Ising model, Phys. Rev. D11, 2098-2103, (1975). doi: 10.1103/PhysRevD.11.2098.

50. R. Balian, J. M. Drouffe, and C. Itzykson, Gauge fields on a lattice 3: Strong coupling expansions and transition points, Phys. Rev. D11, 2104-2119, (1975). doi: 10.1103/ PhysRevD.11.2104. (E) ibid. D11, 2514 (1975), doi: 10.1103/PhysRevD.19.2514.

51. A. Haar, Der Maßbegriff in der Theorie der kontinuierlichen Gruppen, Ann. Math. 34, 147-169, (1933). URL http://www.jstor.org/stable/1968346.

52. F. A. Berezin, The Method of Second Quantization. (Academic, New York, 1966).

53. K. G. Wilson and J. B. Kogut, The renormalization group and the $\epsilon$ expansion, Phys. Rept. 12, 75-200, (1974). doi: 10.1016/0370-1573(74)90023-4.

54. E. Seiler, Gauge theories as a problem of constructive quantum field theory and statistical mechanics, Lect. Notes Phys. 159, 1-192, (1982).

55. K. Osterwalder and R. Schrader, Axioms for Euclidean Green's functions, Commun. Math. Phys. 31, 83-112, (1973). doi: 10.1007/BF01645738.

56. K. Osterwalder and R. Schrader, Axioms for Euclidean Green's functions II, Commun. Math. Phys. 42, 281-305, (1975). doi: 10.1007/BF01608978.

57. K. Osterwalder and E. Seiler, Gauge field theories on the lattice, Ann. Phys. 110, 440-471, (1978). doi: 10.1016/0003-4916(78)90039-8.

58. S. J. Summers. A perspective on constructive quantum field theory. arXiv:1203.3991 [math-ph], (2012).

59. R. Savit, Topological excitations in U(1) invariant theories, Phys. Rev. Lett. 39, 55-58, (1977). doi: 10.1103/PhysRevLett.39.55.

60. T. Banks, R. Myerson, and J. B. Kogut, Phase transitions in Abelian lattice gauge theories, Nucl. Phys. B129, 493-510, (1977). doi: 10.1016/0550-3213(77)90129-8.

61. A. H. Guth, Existence proof of a nonconfining phase in four-dimensional U(1) lattice gauge theory, Phys. Rev. D21, 2291-2307, (1980). doi: 10.1103/PhysRevD.21.2291.

62. J. Fröhlich and T. Spencer, Massless phases and symmetry restoration in Abelian gauge theories and spin systems, Commun. Math. Phys. 83, 411-454, (1982). doi: 10.1007/BF01213610.

63. M. Creutz, Monte Carlo study of quantized SU(2) gauge theory, Phys. Rev. D21, 2308-2315, (1980). doi: 10.1103/PhysRevD.21.2308.

64. M. Creutz, Asymptotic-freedom scales, Phys. Rev. Lett. 45, 313, (1980). doi: 10. 1103/PhysRevLett.45.313.

65. J. B. Kogut, R. B. Pearson, and J. Shigemitsu, Quantum-chromodynamic $\beta$ function at intermediate and strong coupling, Phys. Rev. Lett. 43, 484-486, (1979). doi: 10. 1103/PhysRevLett.43.484.

66. G. Münster, High temperature expansions for the free energy of vortices, respectively the string tension in lattice gauge theories, Nucl. Phys. B180, 23, (1981). doi: 10. 1016/0550-3213(81)90153-X.

67. G. Bhanot and M. Creutz, Variant actions and phase structure in lattice gauge theory, Phys. Rev. D24, 3212, (1981). doi: 10.1103/PhysRevD.24.3212.

68. G. Bhanot, SU(3) lattice gauge theory in four-dimensions with a modified Wilson action, Phys. Lett. B108, 337, (1982). doi: 10.1016/0370-2693(82)91207-2.

69. G. Münster, Strong coupling expansions for the mass gap in lattice gauge theories, Nucl. Phys. B190, 439-453, (1981). doi: 10.1016/0550-3213(81)90570-8.

70. K. Seo, Glueball mass estimate by strong coupling expansion in lattice gauge theories, Nucl. Phys. B209, 200-216, (1982). doi: 10.1016/0550-3213(82)90110-9.

71. J. Smit, Estimate of glueball masses from their strong coupling series in lattice QCD, Nucl. Phys. B206, 309, (1982). doi: 10.1016/0550-3213(82)90537-5. 
72. G. Münster, Physical strong coupling expansion parameters and glueball mass ratios, Phys. Lett. B121, 53, (1983). doi: 10.1016/0370-2693(83)90201-0.

73. J. B. Kogut, D. K. Sinclair, and L. Susskind, A quantitative approach to lowenergy quantum chromodynamics, Nucl. Phys. B114, 199, (1976). doi: 10.1016/ 0550-3213(76)90586-1.

74. T. Banks et al., Strong coupling calculations of the hadron spectrum of quantum chromodynamics, Phys. Rev. D15, 1111, (1977). doi: 10.1103/PhysRevD.15.1111.

75. J. M. Blairon, R. Brout, F. Englert, and J. Greensite, Chiral symmetry breaking in the action formulation of lattice gauge theory, Nucl. Phys. B180, 439, (1981). doi: 10.1016/0550-3213(81)90061-4.

76. J. B. Kogut, A review of the lattice gauge theory approach to quantum chromodynamics, Rev. Mod. Phys. 55, 775-836, (1983). doi: 10.1103/RevModPhys.55.775.

77. H. Kluberg-Stern, A. Morel, and B. Petersson, Spectrum of lattice gauge theories with fermions from a $1 / d$ expansion at strong coupling, Nucl. Phys. B215, 527, (1983). doi: 10.1016/0550-3213(83)90259-6.

78. O. Martin, Mesons and baryons at large $N$ and strong coupling, Phys. Lett. B130, 411, (1983). doi: 10.1016/0370-2693(83)91533-2.

79. L. H. Karsten and J. Smit, Lattice fermions: Species doubling, chiral invariance, and the triangle anomaly, Nucl. Phys. B183, 103, (1981). doi: 10.1016/0550-3213(81) 90549-6.

80. J. B. Kogut and L. Susskind, Hamiltonian formulation of Wilson's lattice gauge theories, Phys. Rev. D11, 395-408, (1975). doi: 10.1103/PhysRevD.11.395.

81. L. Susskind, Lattice fermions, Phys. Rev. D16, 3031-3039, (1977). doi: 10.1103/ PhysRevD.16.3031.

82. N. Kawamoto and J. Smit, Effective Lagrangian and dynamical symmetry breaking in strongly coupled lattice QCD, Nucl. Phys. B192, 100, (1981). doi: 10.1016/ 0550-3213(81)90196-6.

83. H. S. Sharatchandra, H. J. Thun, and P. Weisz, Susskind fermions on a Euclidean lattice, Nucl. Phys. B192, 205, (1981). doi: 10.1016/0550-3213(81)90200-5.

84. J. Smit and J. C. Vink, Renormalized Ward-Takahashi relations and topological susceptibility with staggered fermions, Nucl. Phys. B298, 557, (1988). doi: 10.1016/ 0550-3213(88)90354-9.

85. K. G. Wilson. Quantum chromodynamics on a lattice. In ed. A. Zichichi, New Phenomena in Subnuclear Physics. Plenum, New York, (1977).

86. B. Sheikholeslami and R. Wohlert, Improved continuum limit lattice action for QCD with Wilson fermions, Nucl. Phys. B259, 572-596, (1985). doi: 10.1016/ 0550-3213(85)90002-1.

87. K. Jansen et al., Nonperturbative renormalization of lattice QCD at all scales, Phys. Lett. B372, 275-282, (1996). doi: 10.1016/0370-2693(96)00075-5.

88. M. Lüscher, S. Sint, R. Sommer, and P. Weisz, Chiral symmetry and $\mathrm{O}(a)$ improvement in lattice QCD, Nucl. Phys. B478, 365-400, (1996). doi: 10.1016/ 0550-3213(96)00378-1.

89. R. Frezzotti, P. A. Grassi, S. Sint, and P. Weisz, Lattice QCD with a chirally twisted mass term, JHEP. 0108, 058, (2001).

90. R. Frezzotti and G. C. Rossi, Chirally improving Wilson fermions 1: $\mathrm{O}(a)$ improvement, JHEP. 0408, 007, (2004). doi: 10.1088/1126-6708/2004/08/007.

91. H. B. Nielsen and M. Ninomiya, Absence of neutrinos on a lattice 1: Proof by homotopy theory, Nucl. Phys. B185, 20-40, (1981). doi: 10.1016/0550-3213(81) 90361-8,10.1016/0550-3213(82)90011-6. (E) Nucl. Phys. B195, 541-542, (1982).

92. D. Friedan, A proof of the Nielsen-Ninomiya theorem, Commun. Math. Phys. 85, 
481-490, (1982). doi: 10.1007/BF01403500.

93. P. H. Ginsparg and K. G. Wilson, A remnant of chiral symmetry on the lattice, Phys. Rev. D25, 2649, (1982). doi: 10.1103/PhysRevD.25.2649.

94. Y. Shamir, Chiral fermions from lattice boundaries, Nucl. Phys. B406, 90-106, (1993). doi: 10.1016/0550-3213(93)90162-I.

95. H. Neuberger, Exactly massless quarks on the lattice, Phys. Lett. B417, 141-144, (1998). doi: 10.1016/S0370-2693(97)01368-3.

96. P. Hasenfratz, Lattice QCD without tuning, mixing and current renormalization, Nucl. Phys. B525, 401-409, (1998). doi: 10.1016/S0550-3213(98)00399-X.

97. M. Lüscher, Exact chiral symmetry on the lattice and the Ginsparg-Wilson relation, Phys. Lett. B428, 342-345, (1998). doi: 10.1016/S0370-2693(98)00423-7.

98. S. Chandrasekharan, Lattice QCD with Ginsparg-Wilson fermions, Phys. Rev. D60, 074503, (1999). doi: 10.1103/PhysRevD.60.074503.

99. D. B. Kaplan, A method for simulating chiral fermions on the lattice, Phys. Lett. B288, 342-347, (1992). doi: 10.1016/0370-2693(92)91112-M.

100. R. Narayanan and H. Neuberger, A construction of lattice chiral gauge theories, Nucl. Phys. B443, 305-385, (1995). doi: 10.1016/0550-3213(95)00111-5.

101. M. Lüscher, Abelian chiral gauge theories on the lattice with exact gauge invariance, Nucl. Phys. B549, 295-334, (1999). doi: 10.1016/S0550-3213(99)00115-7.

102. M. Lüscher, Weyl fermions on the lattice and the non-Abelian gauge anomaly, Nucl. Phys. B568, 162-179, (2000). doi: 10.1016/S0550-3213(99)00731-2.

103. M. Creutz, Gauge fixing, the transfer matrix, and confinement on a lattice, Phys. Rev. D15, 1128, (1977). doi: 10.1103/PhysRevD.15.1128.

104. M. Lüscher, Construction of a self-adjoint, strictly positive transfer matrix for Euclidean lattice gauge theories, Commun. Math. Phys. 54, 283, (1977). doi: 10.1007/BF01614090.

105. M. Lüscher and P. Weisz, Definition and general properties of the transfer matrix in continuum limit improved lattice gauge theories, Nucl. Phys. B240, 349, (1984). doi: 10.1016/0550-3213(84)90270-0.

106. M. Di Pierro, An algorithmic approach to quantum field theory, Int. J. Mod. Phys. A21, 405-448, (2006). doi: 10.1142/S0217751X06028965.

107. D. Weingarten, Monte Carlo evaluation of hadron masses in lattice gauge theories with fermions, Phys. Lett. B109, 57-62, (1982). doi: 10.1016/0370-2693(82)90463-4.

108. E. Marinari, G. Parisi, and C. Rebbi, Monte Carlo simulation of the massive Schwinger model, Nucl. Phys. B190, 734-750, (1981). doi: 10.1016/0550-3213(81) 90048-1.

109. C. T. H. Davies et al., High-precision lattice QCD confronts experiment, Phys. Rev. Lett. 92, 022001, (2004). doi: 10.1103/PhysRevLett.92.022001.

110. K. Hagiwara et al., Review of particle physics, Phys. Rev. D66, 010001, (2002). doi: 10.1103/PhysRevD.66.010001.

111. C. Aubin et al., Semileptonic decays of $D$ mesons in three-flavor lattice QCD, Phys. Rev. Lett. 94, 011601, (2005). doi: 10.1103/PhysRevLett.94.011601.

112. I. F. Allison et al., Mass of the $B_{c}$ meson in three-flavor lattice QCD, Phys. Rev. Lett. 94, 172001, (2005). doi: 10.1103/PhysRevLett.94.172001.

113. C. Aubin et al., Charmed meson decay constants in three-flavor lattice QCD, Phys. Rev. Lett. 95, 122002, (2005). doi: 10.1103/PhysRevLett.95.122002.

114. A. Gray et al., The $\Upsilon$ spectrum and $m_{b}$ from full lattice QCD, Phys. Rev. D72, 094507, (2005). doi: 10.1103/PhysRevD.72.094507.

115. D. Besson et al., Improved measurements of $D$ meson semileptonic decays to $\pi$ and $K$ mesons, Phys. Rev. D80, 032005, (2009). doi: 10.1103/PhysRevD.80.032005. 
116. C. Bernard et al., Visualization of semileptonic form factors from lattice QCD, Phys. Rev. D80, 034026, (2009). doi: 10.1103/PhysRevD.80.034026.

117. J. M. Link et al., Measurements of the $q^{2}$ dependence of the $D^{0} \rightarrow K^{-} \mu^{+} \nu$ and $D^{0} \rightarrow \pi^{-} \mu^{+} \nu$ form factors, Phys. Lett. B607, 233-242, (2005). doi: 10.1016/j. physletb.2004.12.036.

118. L. Widhalm et al., Measurement of $D^{0} \rightarrow \pi l \nu(K l \nu)$ form factors and absolute branching fractions, Phys. Rev. Lett. 97, 061804, (2006). doi: 10.1103/PhysRevLett. 97.061804.

119. B. Aubert et al., Measurement of the hadronic form-factor in $D^{0} \rightarrow K^{-} e^{+} \nu_{e}$, Phys. Rev. D76, 052005, (2007). doi: doi:10.1103/PhysRevD.76.052005.

120. S. Dobbs et al., A study of the semileptonic charm decays $D^{0} \rightarrow \pi^{-} e^{+} \nu_{e}, D^{+} \rightarrow$ $\pi^{0} e^{+} \nu_{e}, D^{0} \rightarrow K^{-} e^{+} \nu_{e}$, and $D^{+} \rightarrow \bar{K}^{0} e^{+} \nu_{e}$, Phys. Rev. D77, 112005, (2008). doi: 10.1103/PhysRevD.77.112005.

121. J. Y. Ge et al., Study of $D^{0} \rightarrow \pi^{-} e^{+} \nu_{e}, D^{+} \rightarrow \pi^{0} e^{+} \nu_{e}, D^{0} \rightarrow K^{-} e^{+} \nu_{e}$, and $D^{+} \rightarrow \bar{K}^{0} e^{+} \nu_{e}$ in tagged decays of the $\psi(3770)$ resonance, Phys. Rev. D79, 052010, (2009). doi: 10.1103/PhysRevD.79.052010.

122. A. S. Kronfeld, Twenty-first century lattice gauge theory: Results from the QCD Lagrangian, Annu. Rev. Nucl. Part. Sci. 62, 265-284, (2012). doi: 10.1146/ annurev-nucl-102711-094942.

123. C. Aubin et al., Light hadrons with improved staggered quarks: Approaching the continuum limit, Phys. Rev. D70, 094505, (2004). doi: 10.1103/PhysRevD.70.094505.

124. A. Bazavov et al., Nonperturbative QCD simulations with $2+1$ flavors of improved staggered quarks, Rev. Mod. Phys. 82, 1349-1417, (2010). doi: 10.1103/ RevModPhys.82.1349.

125. S. Aoki et al., 2+1 flavor lattice QCD toward the physical point, Phys. Rev. D79, 034503, (2009). doi: 10.1103/PhysRevD.79.034503.

126. S. Dürr et al., Ab-initio determination of light hadron masses, Science. 322, 12241227, (2008). doi: 10.1126/science.1163233.

127. W. Bietenholz et al., Flavour blindness and patterns of flavour symmetry breaking in lattice simulations of up, down and strange quarks, Phys. Rev. D84, 054509, (2011). doi: 10.1103/PhysRevD.84.054509.

128. N. H. Christ et al., The $\eta$ and $\eta^{\prime}$ mesons from lattice QCD, Phys. Rev. Lett. 105, 241601, (2010). doi: 10.1103/PhysRevLett.105.241601.

129. J. J. Dudek et al., Isoscalar meson spectroscopy from lattice QCD, Phys. Rev. D83, 111502, (2011). doi: 10.1103/PhysRevD.83.111502.

130. E. B. Gregory, A. C. Irving, C. M. Richards, and C. McNeile, A study of the $\eta$ and $\eta^{\prime}$ mesons with improved staggered fermions, Phys. Rev. D86, 014504, (2012). doi: 10.1103/PhysRevD.86.014504.

131. C. Bernard et al., Tuning Fermilab heavy quarks in $2+1$ flavor lattice QCD with application to hyperfine splittings, Phys. Rev. D83, 034503, (2011). doi: 10.1103/ PhysRevD.83.034503.

132. E. B. Gregory et al., Precise $B, B_{s}$, and $B_{c}$ meson spectroscopy from full lattice QCD, Phys. Rev. D83, 014506, (2011). doi: 10.1103/PhysRevD.83.014506.

133. D. Mohler and R. M. Woloshyn, $D$ and $D_{s}$ meson spectroscopy, Phys. Rev. D84, 054505, (2011). doi: 10.1103/PhysRevD.84.054505.

134. Z. Fodor and C. Hoelbling, Light hadron masses from lattice QCD, Rev. Mod. Phys. 84, 449-495, (2012). doi: 10.1103/RevModPhys.84.449.

135. J. Beringer et al., Review of particle physics, Phys. Rev. D86, 010001, (2012). doi: 10.1103/PhysRevD.86.010001.

136. C. J. Morningstar and M. J. Peardon, The glueball spectrum from an anisotropic 
lattice study, Phys. Rev. D60, 034509, (1999). doi: 10.1103/PhysRevD.60.034509.

137. C. M. Richards, A. C. Irving, E. B. Gregory, and C. McNeile, Glueball mass measurements from improved staggered fermion simulations, Phys. Rev. D82, 034501, (2010). doi: 10.1103/PhysRevD.82.034501.

138. J. M. Bulava et al., Excited state nucleon spectrum with two flavors of dynamical fermions, Phys. Rev. D79, 034505, (2009). doi: 10.1103/PhysRevD.79.034505.

139. R. G. Edwards, J. J. Dudek, D. G. Richards, and S. J. Wallace, Excited state baryon spectroscopy from lattice QCD, Phys. Rev. D84, 074508, (2011). doi: 10.1103/PhysRevD.84.074508.

140. V. D. Burkert, The $N^{*}$ physics program at Jefferson Lab, Chin. Phys. C33, 10431050, (2009). doi: 10.1088/1674-1137/33/12/001.

141. K. J. Juge, J. Kuti, and C. Morningstar, Fine structure of the QCD string spectrum, Phys. Rev. Lett. 90, 161601, (2003). doi: 10.1103/PhysRevLett.90.161601.

142. K. J. Juge, J. Kuti, and C. Morningstar. QCD string formation and the Casimir energy. In eds. H. Suganuma et al., Confinement 2003, pp. 233-248, Singapore, (2004). World Scientific.

143. M. Lüscher, G. Münster, and P. Weisz, How thick are chromoelectric flux tubes?, Nucl. Phys. B180, 1, (1981). doi: 10.1016/0550-3213(81)90151-6.

144. G. S. Bali, H. Neff, T. Düssel, T. Lippert, and K. Schilling, Observation of string breaking in QCD, Phys. Rev. D71, 114513, (2005). doi: 10.1103/PhysRevD.71. 114513.

145. J. Greensite, The confinement problem in lattice gauge theory, Prog. Part. Nucl. Phys. 51, 1-83, (2003). doi: 10.1016/S0146-6410(03)90012-3.

146. A. Duncan, E. Eichten, and H. Thacker, Lattice QCD, the quark model, and heavylight wave functions, Phys. Lett. B303, 109-112, (1993). doi: 10.1016/0370-2693(93) 90052-J.

147. Y. Nambu, Axial vector current conservation in weak interactions, Phys. Rev. Lett. 4, 380-382, (1960). doi: 10.1103/PhysRevLett.4.380.

148. J. Goldstone, Field theories with superconductor solutions, Nuovo Cim. 19, 154-164, (1961). doi: 10.1007/BF02812722.

149. H. Fukaya et al., Determination of the chiral condensate from QCD Dirac spectrum on the lattice, Phys. Rev. D83, 074501, (2011). doi: 10.1103/PhysRevD.83.074501.

150. N. Ishii, S. Aoki, and T. Hatsuda, The nuclear force from lattice QCD, Phys. Rev. Lett. 99, 022001, (2007). doi: 10.1103/PhysRevLett.99.022001.

151. S. R. Beane et al., Light nuclei and hypernuclei from quantum chromodynamics in the limit of SU(3) flavor symmetry. (2012). arXiv:1206.5219 [hep-lat].

152. B. Carazza and H. Kragh, Heisenberg's lattice world: The 1930 theory sketch, Am. J. Phys. 63, 595-605, (1995). doi: 10.1119/1.17848.

153. W. Heisenberg, Die Selbstenergie des Elektrons, Z. Phys. 65, 4-13, (1930). doi: 10.1007/BF01397404.

154. H. Kragh, Arthur March, Werner Heisenberg, and the search for a smallest length, Revue d'Histoire des Sciences. 48(4), 401-434, (1995). doi: 10.3406/rhs.1995.1239.

155. C. T. Hill. private communication.

156. O. P. Pedersen and T. Bundgaard. The birth of SOMA? URL http://www. fam-bundgaard.dk/SOMA/NEWS/N030310.HTM. (Retrieved August 2012).

157. M. Gardner, The Second Scientific American Book of Mathematical Puzzles and Diversions. (University of Chicago, Chicago, 1987). 